EPJ Web of Conferences 20, 05004 (2012)

DOI: $10.1051 /$ epjconf/20122005004

(C) Owned by the authors, published by EDP Sciences, 2012

\title{
Research and development of a CBM-RICH prototype
}

\author{
Jungyu Yi, ${ }^{\text {, }}$, Jongsik Eum, Kunsu Oh, Jihye Song, and In-Kwon Yoo \\ Department of Phyics, Pusan National University, 609-735 Pusan, Republic of Korea.
}

\begin{abstract}
We have developed a gaseous Ring Imaging CHerenkov detector prototype (PNU-RICH2) of the CBM-RICH detector. This prototype has the same radiator length as the planned CBM-RICH detector. The PNU-RICH2 includes a spherical concave mirror and 2 types of Multi-anode PMTs. The PNU-RICH2 detector has tested at the PAL-TEST LINAC with $60 \mathrm{MeV}$ electron beam. A detector performance from the beam test will be presented and discussed in comparison with simulation results.
\end{abstract}

\section{Introduction}

A Ring Imaging CHerenkov (RICH) detector is widely used for identifying particles, which fly through the specific medium with a speed higher than the speed of light in the medium $(c / n, n$ : refractive index), and produce the Cherenkov radiation[1,2]. A RICH detector in general consists of the vessel containing Cherenkov radiator, a concave reflector and photo detectors at focal plane of the reflector. One of the great advantages of this Ring Imaging technique is, one can easily change the measurable particle momentum range with changing the Cherenkov radiator with various refractive index. However, since the produced Cherenkov photon has to transmitted through the Cherenkov medium itself and its amount is inversely proportional to the wavelength squared, the detected photon number is extremely low and thus special consideration on the reflectors and radiators is required to keep the UV-photons onto the photo detector as much as possible[3].

The Heavy Ion Physics Experiment (HIPEx) group in Pusan National University is now participating the RICH R\&D for the Compressed Baryonic Matter (CBM) experiment at Facility of Antiproton and Ion Research (FAIR) in GSI, Germany[4]. The CBM experiment project is now on the R\&D stage for the detection of various particles produced in heavy-ion collisions from 8 to $45 \mathrm{~A} \mathrm{GeV}$ beam energy. The matter created in this collision covers the intermediate range of the QCD phase diagram. In this range, a phase transition between hadronic and partonic matter and the onset of chiral symmetry restoration are expected. An experimental confirmation of these phase boundaries would be of fundamental interest for a better understanding of the strong interaction. The CBM RICH detector needed for an efficient and clean electron identification for $p \leq 8 \mathrm{GeV} / c$ in a wide phase space acceptance and with pion suppression in the order of 500-1000.

\section{Development of a CBM-RICH prototype}

According to the experimental requirements of the CBM-RICH to identify high momentum electrons, it is designed to use a gas radiator or gas mixtures. For such gas radiator research, therefore, one needs to develop not only a prototype with full components, but also a gas system, which stably provides the radiator gas with controlling and monitoring. In this paper, we present development of a mini-RICH detector and the PNU-RICH2 manufactured by Pusan HIPEx group as a CBM-RICH prototype and its test status in Pohang Accelerator Laboratory (PAL) with a Gas Control System (GCS) consisting of PLC and PVSS.

\footnotetext{
a e-mail: fireplay@pusan.ac.kr
}

This is an Open Access article distributed under the terms of the Creative Commons Attribution-Noncommercial License 3.0, which permits unrestricted use, distribution, and reproduction in any noncommercial medium, provided the original work is properly cited. 


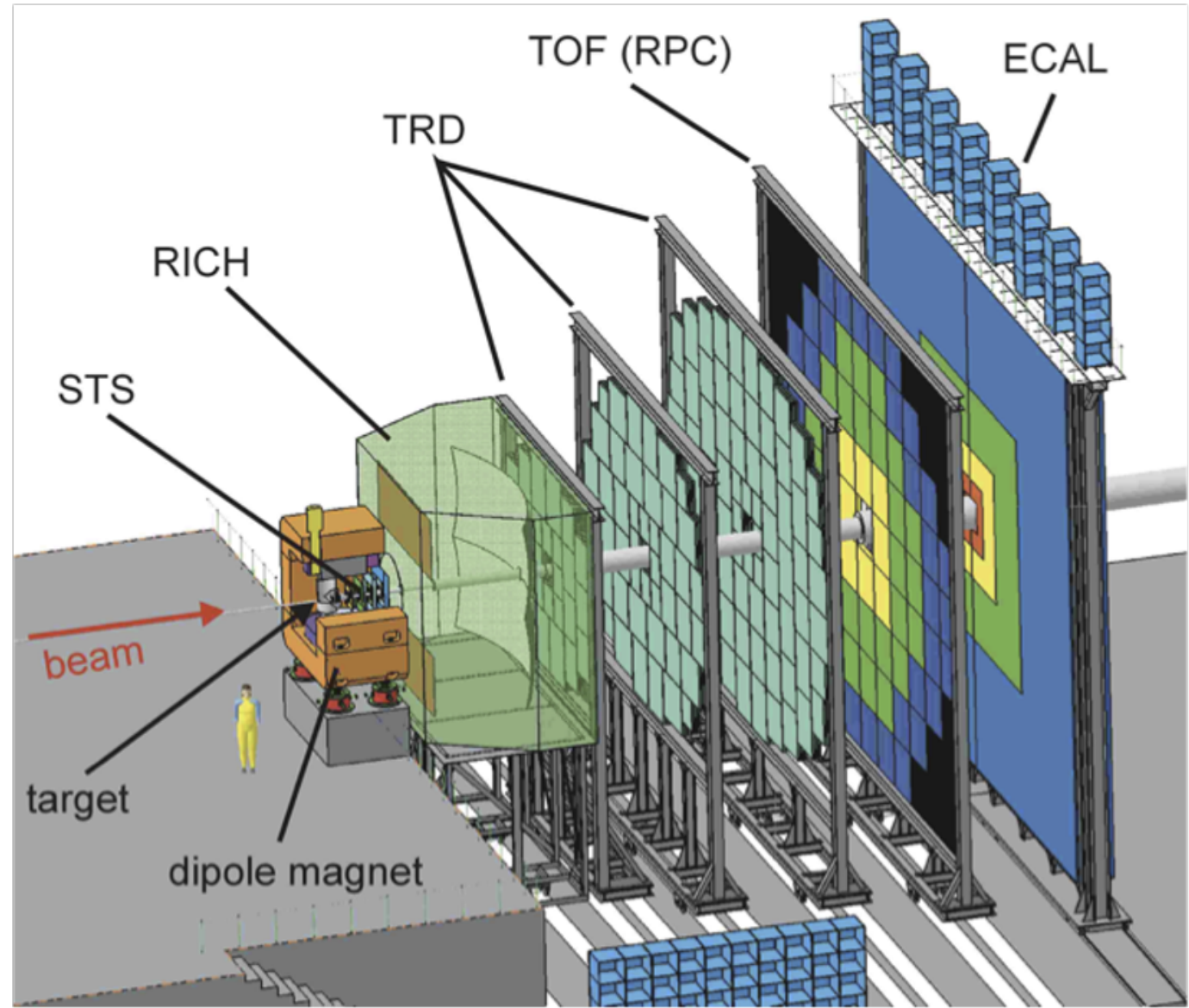

Fig. 1. Compressed Baryonic Matter (CBM) at FAIR, GSI

\subsection{Development of a mini-RICH detector}

The developed mini-RICH detector[5] is shown schematically in Figure 2. The detector vessel is constructed as a rectangular box with $10 \mathrm{~mm}$ thick aluminium walls, having inner dimensions of $600 \times$ $400 \times 200 \mathrm{~mm}^{3}$. The vessel serves as a gas container as well as a darkroom shielding the MAPMT from outside light. The electron beam enters through a Mylar window of $3 \mathrm{~mm}$ diameter. A $25.4 \mathrm{~mm}$ diameter spherical silver coated aluminium mirror with curvature $R=750 \mathrm{~mm}$ is located at a radiator length $L=500 \mathrm{~mm}$ and rotated by $\alpha=5^{\circ}$. A 64-pixel MAPMT is centered at $\alpha=10^{\circ}$ in the focal plane at $375 \mathrm{~mm}$ distance from the mirror.

Figure 3 shows the performance of the mini-RICH detector in the electron beam test. One clearly sees an enhancement of QDC values in the expected ring region in comparison with the outer regions by a factor of approximately 1.4. But, the ring image was smeared because of the electron beam at PAL was rather wide, unstable and unfocussed such that the ring was not always at the same position.

\subsection{Development of the PNU-RICH2 detector}

In order to improve Cherenkov light detection of the mini-RICH detector and to investigate properties of the future CBM-RICH detector, a vessel of $1.76 \mathrm{~m}$ radiator length was prepared following the layout 
Hadron Nuclear Physics (HNP) 2011

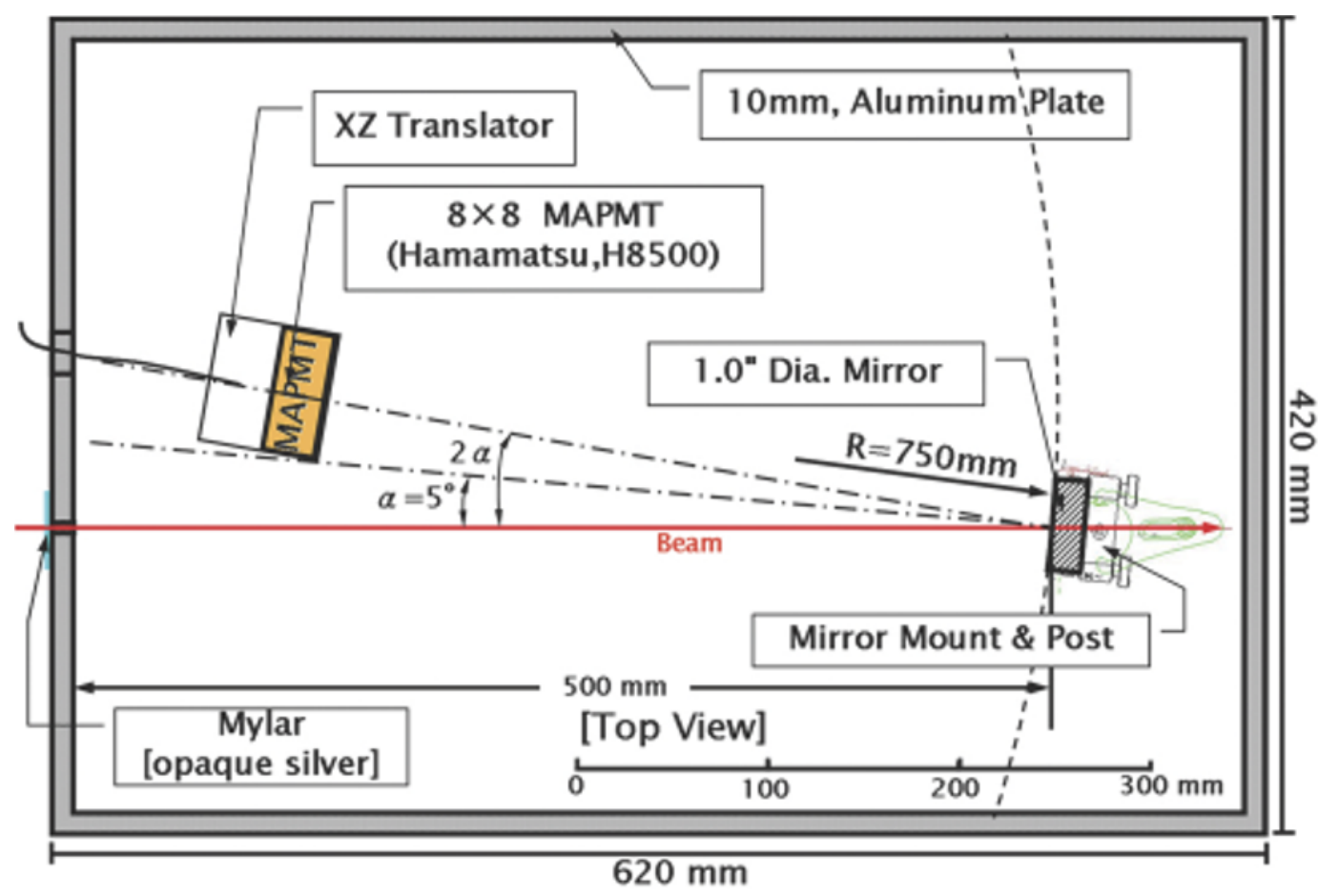

Fig. 2. Schematical drawing of mini-RICH (top view)

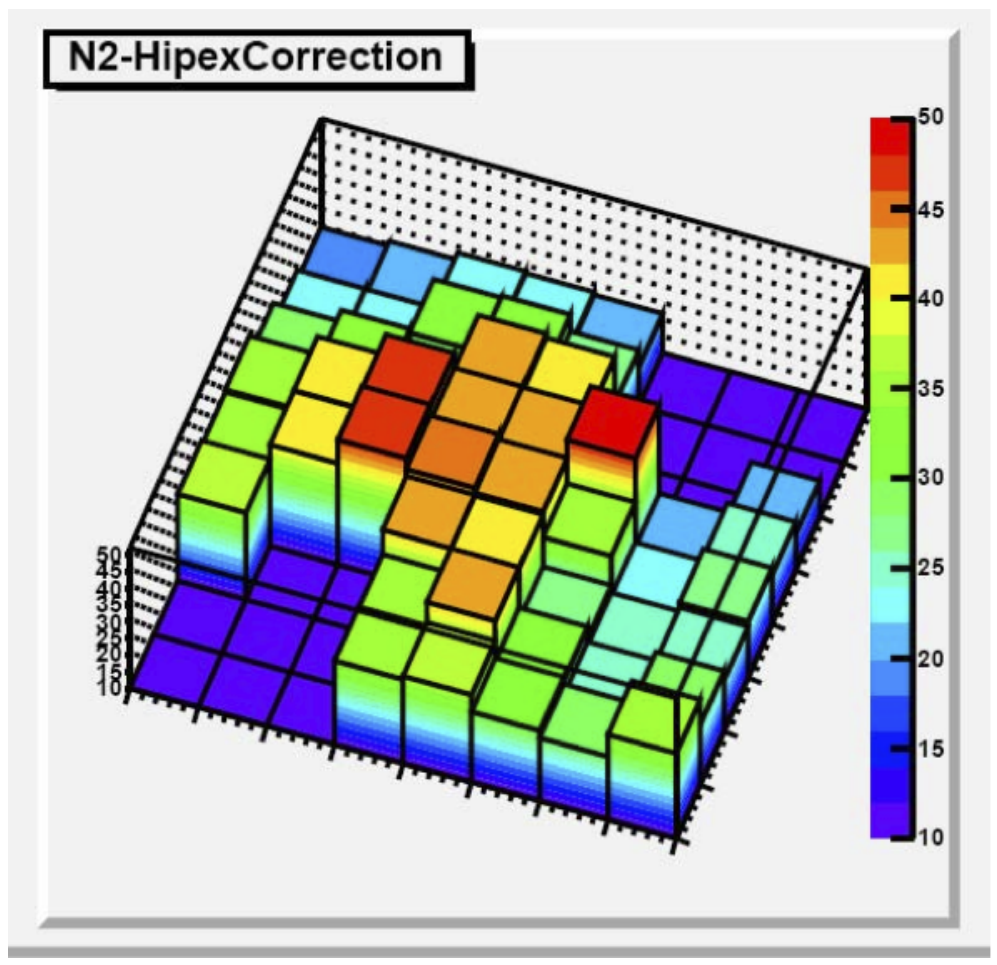

Fig. 3. Test result of mini-RICH with $N_{2}$ gas, $60 \mathrm{MeV}$ electrons 


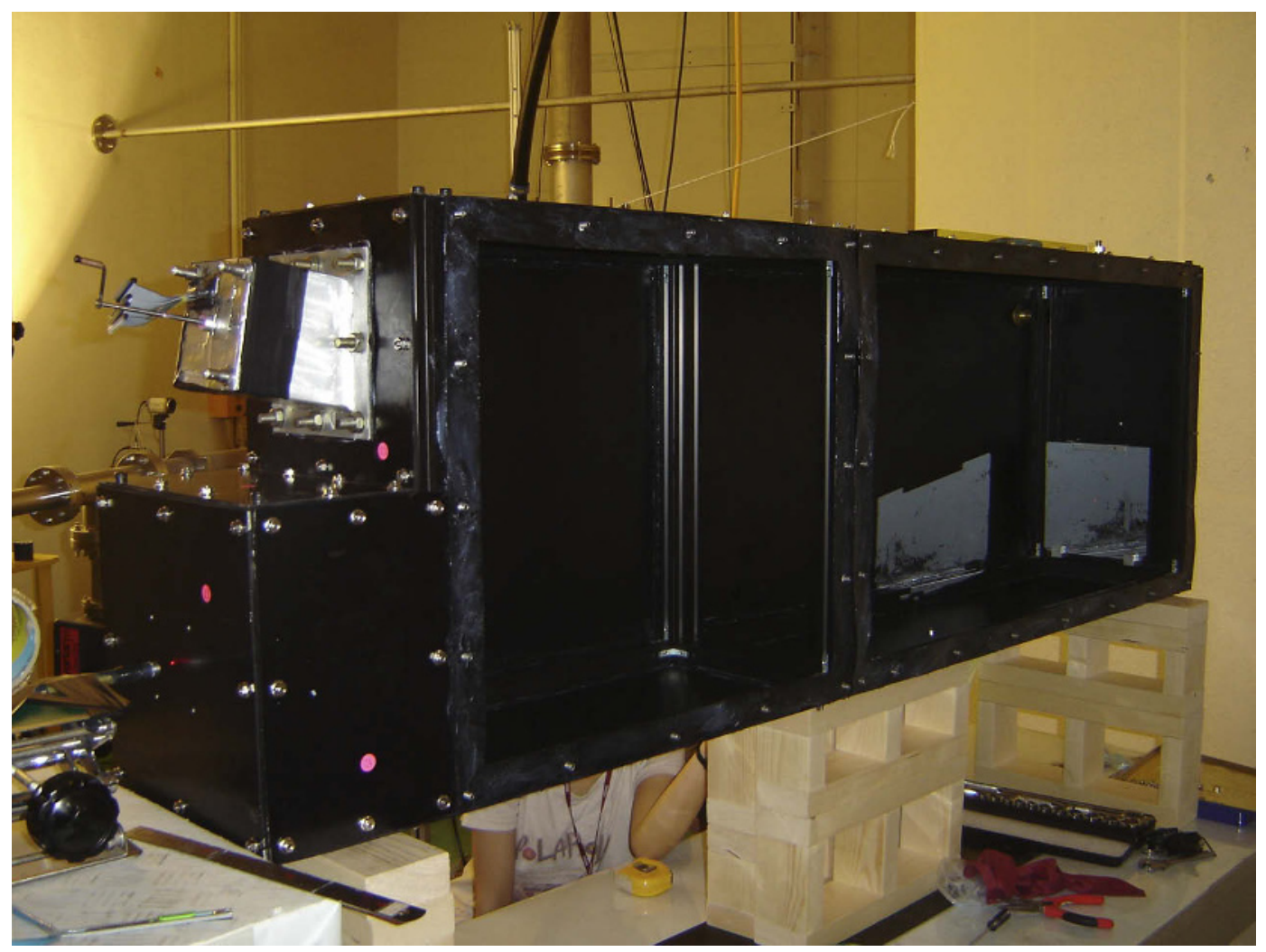

Fig. 4. PNU-RICH2 : CBM-RICH prototype in Pusan.

proposal of the CBM-RICH detector[7]. The detector vessel is constructed with $2 \mathrm{~mm}$ thick anodized aluminum panels which are supported by an aluminum frame having dimensions of about $1.8 \times 0.6 \times 0.3$ $\mathrm{m}^{3}$. The electron beam enters through a $25 \mu \mathrm{m}$ thick kapton foil window of $1 \mathrm{~mm}$ diameter. For light reflection and focusing onto the photodetector a spherical concave mirror (first CBM mirror prototype from FLABEG company) of $20 \times 20 \mathrm{~cm}^{2}$ size and radius of curvature $3.2 \mathrm{~m}$ is positioned at a radiator length $\mathrm{L}=1.76 \mathrm{~m}$ in the vessel. Its alignment is precisely adjustable in 2 rotational axes with micrometer actuators. The CBM-mirror is coated with $\mathrm{MgF}_{2}$ that has shown efficient reflection of UV-photons[6].

Two types of Multi-anode PMTs (MAPMTs) from Hamamatsu have been implemented: one with borosilicate window (H8500) and another with a UV extended window (H8500C-03). The latter MAPMT has $\sim 20 \%$ quantum efficiency in the UV region down to $200 \mathrm{~nm}$ wavelength. Thus the photo detection with H8500C-03 is strongly enhanced compared to the H8500. The two of 64 channel MAPMTs are installed at diagonal positions within the PMT mount box that has space for 4 MAPMTs in total. This mount box can adjust MAPMTs at various focal planes within a $\pm 50 \mathrm{~mm}$ range. This flexibility has been planned to enable us to cover unexpected deviations of curvature of mirrors. A comparison of the new PNU-RICH2 and the first mini-RICH is summarized in Table 1.

\section{Performance Test with $60 \mathrm{MeV}$ electrons}

The PNU-RICH2 detector performance was tested with $60 \mathrm{MeV}$ electrons at the Test LINAC beam line of the Pohang Accelerator Laboratory (PAL), Pohang, Korea. The conceptual picture of experimental setup is shown in Figure 5. 
Table 1. Parameters for the mini-RICH and the PNU-RICH2 detector.

\begin{tabular}{lll}
\hline & mini-RICH & PNU-RICH2 \\
\hline \hline Radiator length $(L[\mathrm{~m}])$ & 0.55 & 1.76 \\
\hline No. of photons produced for $L$ & $\sim 11$ /electron & $\sim 34 /$ electron \\
\hline No. of PMT channels & 64 & $128(256)$ \\
\hline Curvature of Mirror $(\mathrm{R}[\mathrm{m}])$ & 0.75 & 3.2 \\
\hline Mirror reflectivity & $\geq 85 \%, \lambda \geq 380 \mathrm{~nm}$ & $\geq 80 \%, \lambda \geq 200 \mathrm{~nm}$ \\
\hline Radiator & Dry Air, $\mathrm{N}_{2}$ & $\mathrm{~N}_{2}, \mathrm{CO}_{2} \&$ mixed gas \\
\hline Calculated ring diameter $[\mathrm{mm}]$ & $17.16\left(\mathrm{~N}_{2}\right)$ & $73.21\left(\mathrm{~N}_{2}\right), 91.91\left(\mathrm{CO}_{2}\right)$ \\
\hline
\end{tabular}

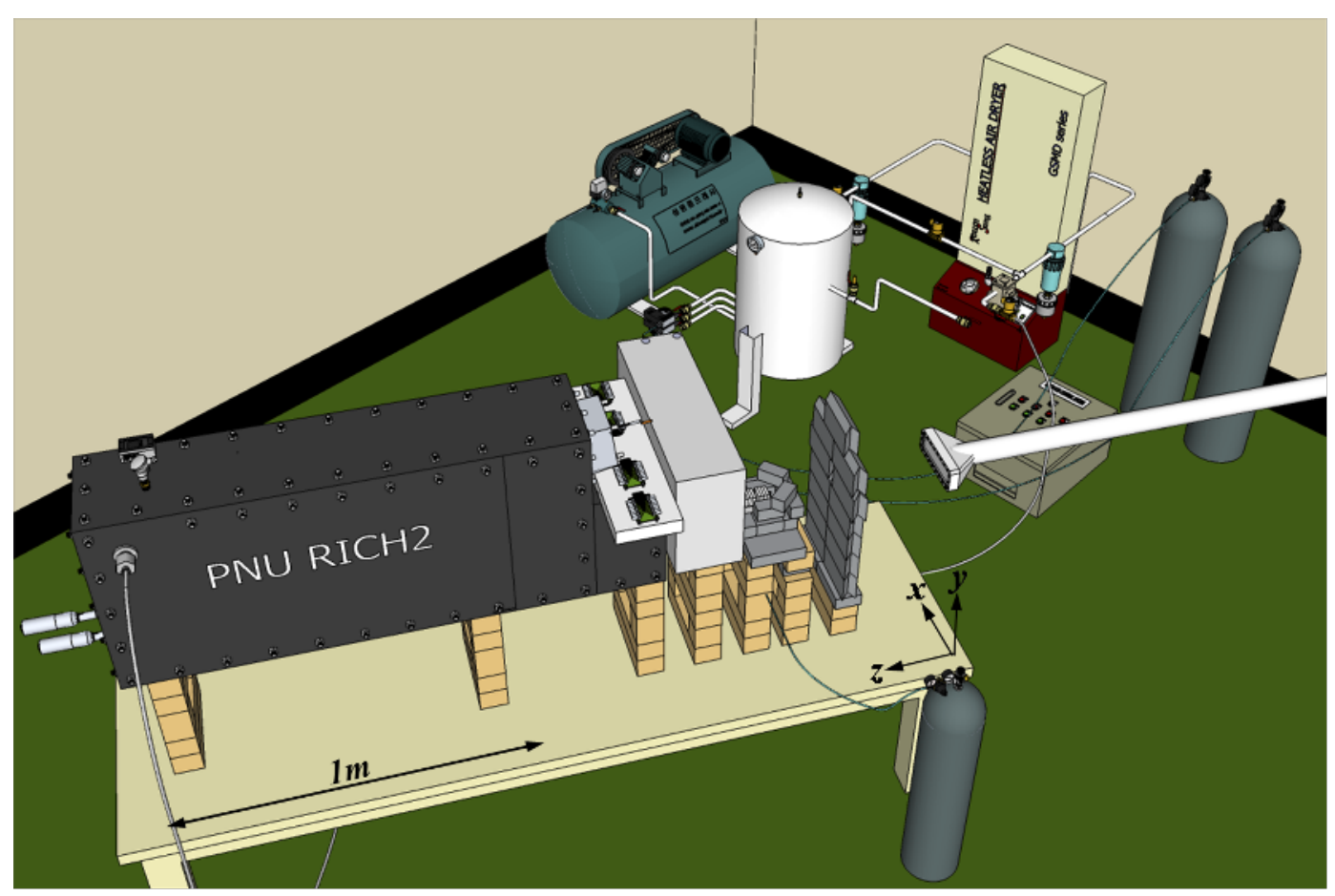

Fig. 5. Conceptual picture of experimental setup with gas system at Test LINAC, PAL.

\subsection{Experimental setup}

The center of PNU-RICH2 prototype detector was aligned to the center of collimator hall behind a beam window. Two scintillation detectors are installed in front and back side of the PNU-RICH2 vessel for validation of electron path. Additionally we used a Multi-Wire Proportional Chamber (MWPC) of $64 \times 56 \mathrm{~mm}^{2}$ with 32 cathode pads of $16 \times 7 \mathrm{~mm}^{2}$ for measuring the beam size at the front of the vessel. 


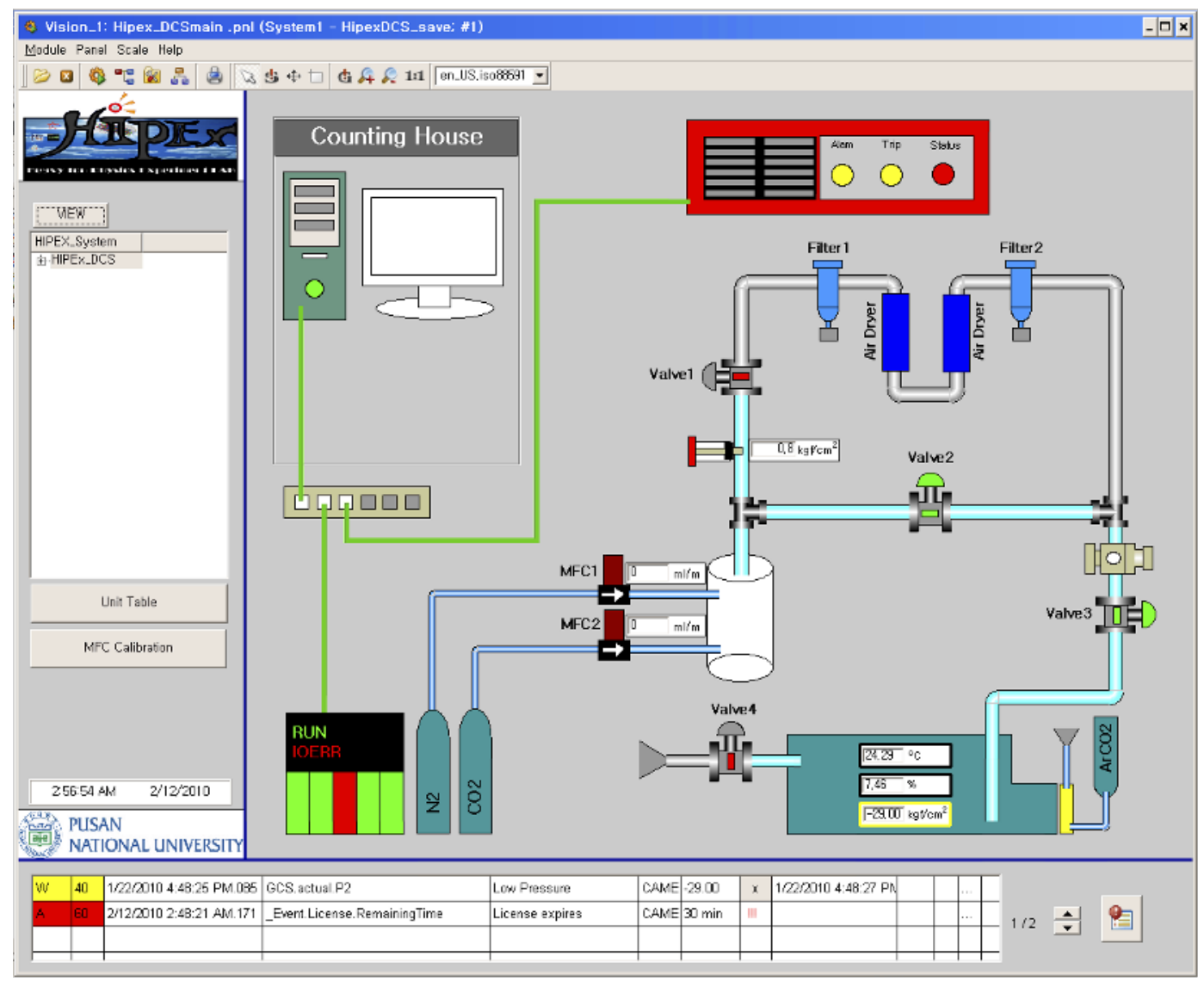

Fig. 6. DCS User Interface.

\subsection{Gas Monitoring and Control System}

A Programmable Logic Controller (PLC) is installed with sensors and controllers for gas monitoring and control. Mass flow controllers and electric solenoidal valves are used for gas flow rate control and to manage the flow directions. We can control gas flow up to $10 \mathrm{~L} / \mathrm{min}$. and make various gas mixtures with the MFC. The sensors are also installed at the vessel to monitor the gas conditions. The temperature, humidity and pressure can be monitored by using a $\mathrm{TH}$ sensor with accuracies $\pm 0.3^{\circ} \mathrm{C}$ and $\pm 1.8 \%$ R.H, the Pressure transmitters up to $5 \mathrm{kgf} / \mathrm{cm}^{2}$ in the vessel and pipes with accuracy $\pm 0.3 \%$ F.S., respectively.

For online monitoring and control of the gas system and also of the High Voltage (HV) supply system, we use a control software tool, PVSSII adapted in LHC experiments. Its interface and simple test are illustrated in Figure 6 and 7.

\subsection{Test Results and Comparison with simulation}

The performance test of the PNU-RICH2 with $60 \mathrm{MeV}$ electron is shown in Figure 8 (a) with an expected ring size calculated for the $\mathrm{N}_{2}$-radiator (see in Table 1). Since experimental data shows no ring image on the photo detection plane, it was compared to the results integrated over $10^{6}$ events from the simulation performed in cbmroot framework (Figure 8 (b)) : A $10 \mathrm{~cm}$ thick lead wall with a $1 \mathrm{~mm}$ 


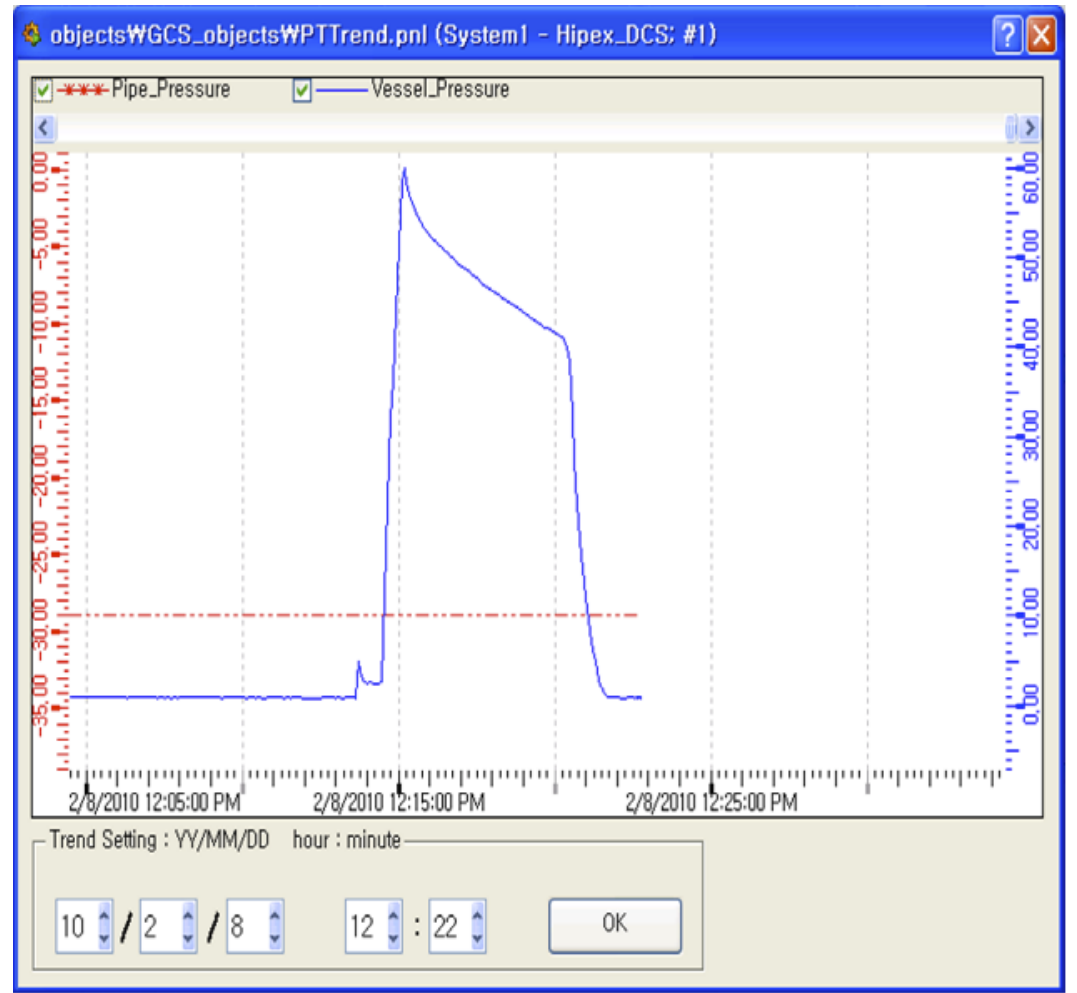

Fig. 7. DCS User Interface and Monitoring test.

hole is set in front of the PNU-RICH2 with $1.76 \mathrm{~m}$ long $\mathrm{N}_{2}$ gas radiator. The $60 \mathrm{MeV}$ electron beam size and possible scattering polar angle are set to $2 \times 2 \mathrm{~mm}^{2}$ and $2^{\circ}$ at the starting point, respectively in the simulation.

The Figure 9 is a single event display of the simulation results with the same conditions. Although any ring image is observed neither in the experimental data nor in the simulation results integrated over $10^{6}$ events as shown in Figure 8, a single event display is showing a possibility to observe a single ring image, which is not always centered at any fixed positions of photo detection plane. In comparison with single $60 \mathrm{MeV} / \mathrm{c}$ electron simulation, the PNU-RICH2 detection feasibility is studied with 1000 events of $1.5 \mathrm{GeV} / \mathrm{c}$ electrons as well, since the Test LINAC in PAL provides no single electron measurement. As shown in Figure 10 for the simulation results, higher energy electrons are not much scattered and mostly fly straightforward along the expected path, with resulting nice ring images even for 1000 events.

Finally one concludes, either each event (electron) must be resolved and recorded as a ring image with simultaneous data acquisition, or higher energy electrons can be used for enhanced ring image measurement even for integrated events. For this condition, reduction of the beam intensity and size is required as much as possible with using secondary electrons scattered at a proper target, and an enhanced data acquisition techniques and devices like n-XYTERs must be taken for the test experiment. Alternatively higher energy electron beam facility can be considered to be used.

\section{Summary and Outlook}

As the R\&D step for the CBM-RICH, a detector prototype PNU-RICH2 is constructed with comparable geometrical dimensions to the current CBM-RICH design in [7] for performance tests of different gas radiators and the determination of requirements on stability of various gas parameters. Additionally 


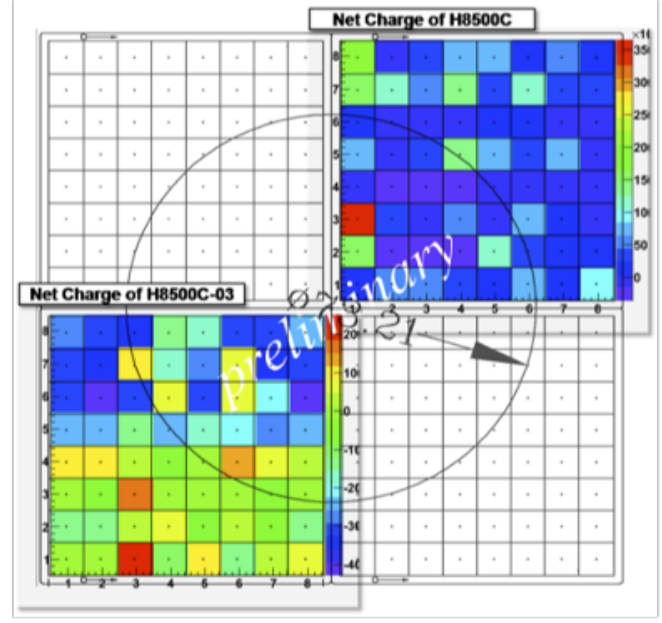

(a)

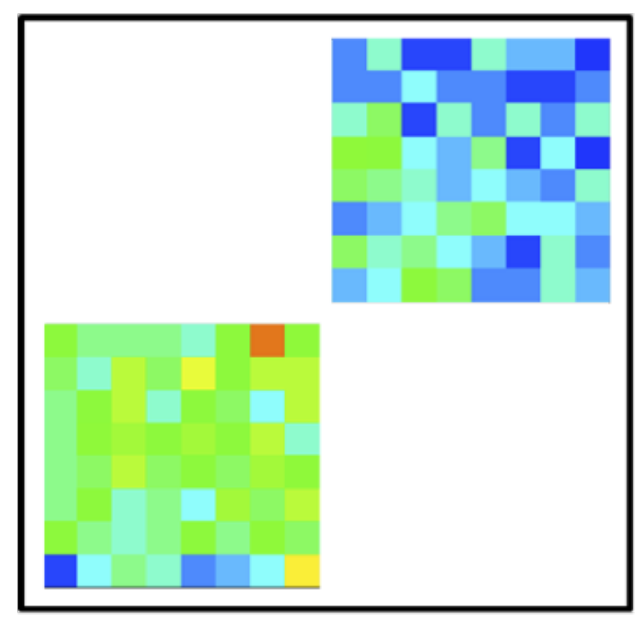

(b)

Fig. 8. (a) Performance test results and a calculated ring size on the 2 MAPMTs plane consisting of $8 \times 8$ channels each. Upper right MAPMT is H8500, and bottom left MAPMT is H8500C-03. (b) Simulation results for $10^{6}$ events from the same experimental conditions.

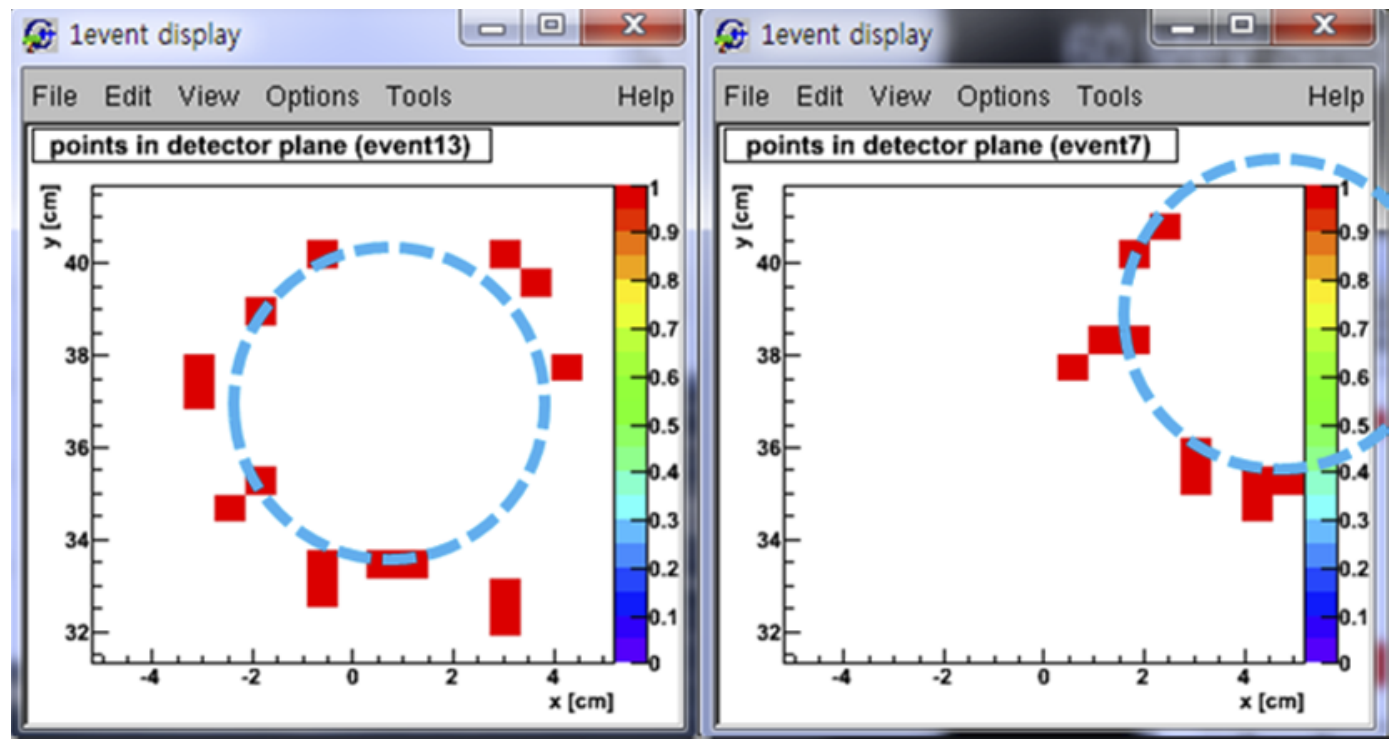

Fig. 9. A single event display of the simulation results with the same experimental conditions.

a gas monitoring and control system based on PVSSII and simulation study with cbmroot framework are successfully progressed.

Performance test was not successful, however, due to scattered electrons of high intensity and low energy electron beam, which never enabled us to resolve single event. As learned from test experiment and simulation study, beam properties with reducing its intensity have to be studied in detail for a successful operation of the RICH prototype. Furthermore an enhanced data acquisition system (CBM$\mathrm{XYTER}$ ) is being expected to be available in near future. 


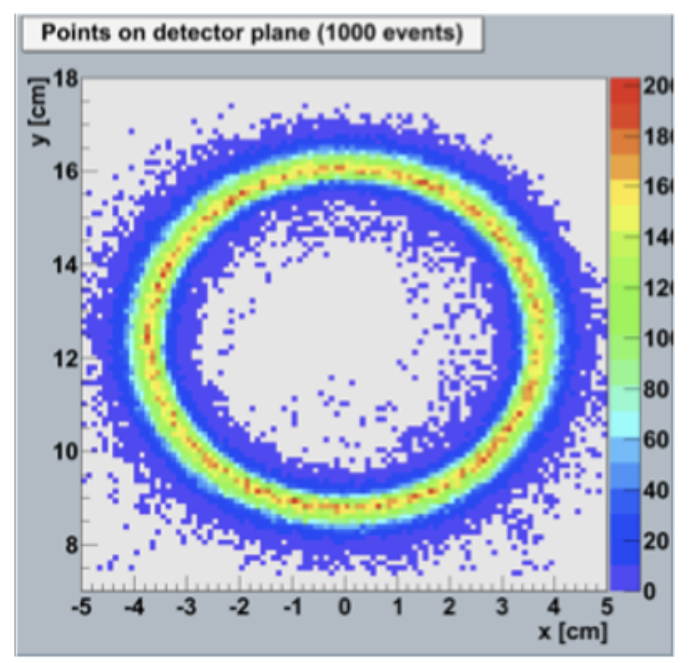

(a)

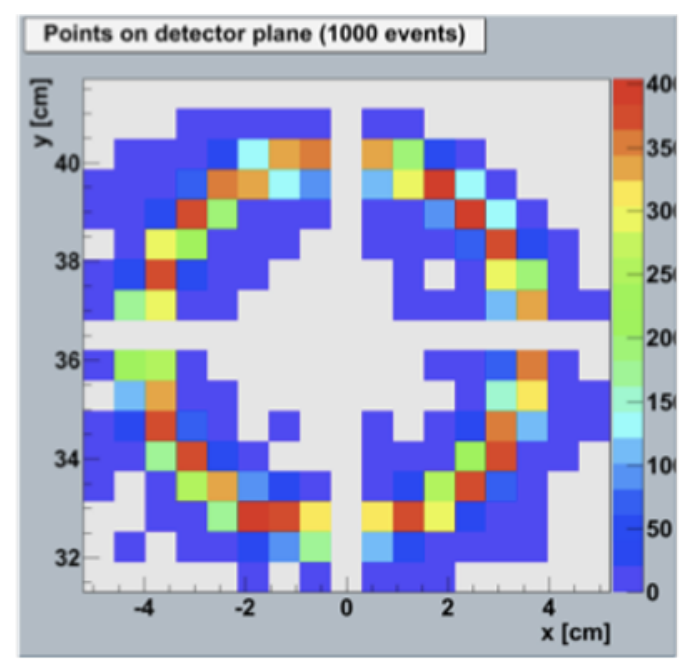

(b)

Fig. 10. 1000 events of $1.5 \mathrm{GeV}$ electron results of the simulation with the same experimental conditions : (a) the Cherenkov photons arriving at Photo detector plane. (b) Signals detected by $16 \times 16$ anode PMT channels.

\section{Acknowledgment}

The work was supported for two years by Pusan National University Research Grant.

\section{References}

1. P.A. Cherenkov Dokl. Akad. Nauk SSSR 2 (1934) 451

2. P.A. Cherenkov Dokl. Akad. Nauk SSSR 21 2(1938) 117

3. I.E. Tamm and I.M. Frank Dokl. Akad. Nauk SSSR 214 (1937) 107

4. CBM collaboration, Compressed Baryonic Matter Experiment Technical Status Report (CBM experiment, February 2005)

5. J.G. Yi et al., CBM Progress Report 2008, (2009), 22

6. M. Dürr, A. Braem and C. Höhne, FAIR-EXPERIMENTS-15, GSI Scientific Report 2008

7. C. Höhne et al., NIM A 595 (2008) 187 